Thomas J. Matula, Ph.D.

Applied Physics Laboratory

1013 NE 40th St.

Seattle, WA 98105

September 17, 1997

Ramoncita N. Massey

U.S. Department of Energy

EM-64/Forrestal Building

Washington D.C. 20585

Dear Ms. Massey

Enclosed is our yearly report on the EMSP project on the sonophysics and sonochemistry of liquid waste quantification and remediation. I feel that we've made excellent progress. We have performed three tasks, and found that only one task didn't perform as we had hoped. These tasks are outlined here:

- We attempted to quantify the electrical signals from cavitation bubbles, and found that previous signals observed may simply be due to direct impact of cavitation bubbles on the probe. We found no electrical pulses. This means that the thermal mechanism for sonochemical activity may be the principle mechanism involved; however, we also found that the theory of cooperative effects (the analogous effect of lasing may be useful) does predict some effects that we've observed. We've submitted a manuscript on this effect for publication.

-We attempted to measure the near-IR spectra from sonoluminescence in cavitation fields, but have had difficulty in getting reliable results.

-We have successfully increased the light-emission from sonoluminescence in cavitation fields by a few orders of magnitude by using what we call "pressure jumping." This may be an extremely important discovery, and we are now looking at the increases in sonochemical degradation rates using this system.

If you have any questions or comments, please don't hesitate to call me.

Sincerely

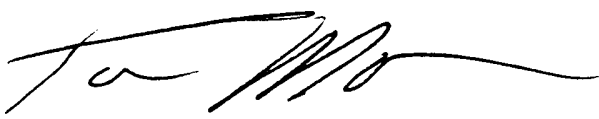

Thomas J. Matula 


\title{
THE SONOPHYSICS AND SONOCHEMISTRY OF LIQUID WASTE QUANTIFICATION AND REMEDIATION
}

\author{
THOMAS J. MATULA, PI
}

\section{YEAR 1 REPORT}

This report constitutes a summary of our activity with reference to grant \#DE-FG07-96ER62310.

Our first year has been extremely successful. We were able to begin our studies immediately, without having to wait for equipment, etc. This report gives details on three projects that were initiated during the first year.

The first project to be described involves an experiment to determine the basic mechanism for cavitation-chemistry, or sonochemistry, in particular the light-emission process: What is the fundamental mechanism for light emission from cavitating bubbles? There are many theories, but most fall into one of two camps. The first is that during the bubble collapse, the interior heats up quazi-adiabatically, and light emission is a result of an incandescence. The second camp claims that the light-emission process is electrical. During the bubble collapse, there is an electrical discharge that results in light emission. Chemical degradation optimization depends greatly on which mechanism is dominant. We proposed to look for an electrical signal that would be generated if discharges were involved.

We designed and built two cavitation systems to explore the electrical pulse hypothesis. In addition, we designed and built several probes to detect an electrical pulse. The probes were differential probes, since a single probe acts as an antenna. We hoped to remove a majority of the common mode signals using the differential probe. A schematic of the probe is illustrated below. Each probe consisted of a set of insulated 32-gauge copper wire surrounded by a l-mm hollow copper tube that was grounded to the rest of the system. The probe tips were separated by approximately $0.5 \mathrm{~mm}$. The probes were connected to a commercial high speed differential amplifier with a gain of 10 . The output was then sent to an oscilloscope for recording the data.

Fig. 1. A schematic of the differential probe used to look for electrical signals from a cavitation field. The probe consists of two insulated 32-gauge wires connected to a differential amplifier (INA106KP-ND). A third wire, connected to a hollow copper tube surrounding the probe tips, was used as a common signal ground. The output of the x10 differential amp was sent to an oscilloscope for recording and analyzing the signal. The diameter of the system was approximately $1 \mathrm{~mm}$. Silver paint was used to coat the tips, except at the extreme edges, to decrease the noise level.

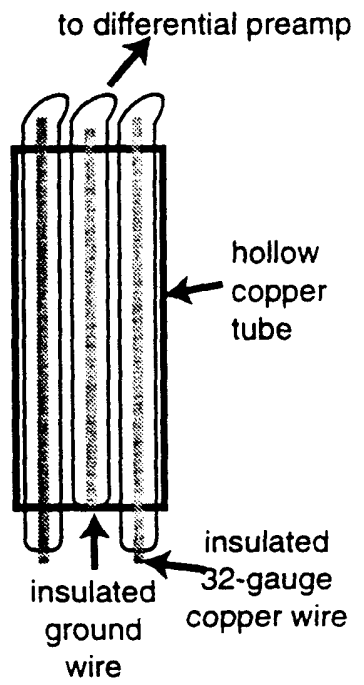


A similar probe was constructed to observe an electrical signal (if any) from a single cavitating bubble; however, in order to position the probe close to the bubble, the probe tips were oriented such that the tips faced each other, with a separation of approximately $1 \mathrm{~mm}$.

Our experiments showed that one does get an electrical signal from a cavitation field: however the probe must be in contact with the field in order to detect the signal. Figure 2 illustrates the electrical pulse we receive from a probe that comes in contact with a well-defined cavitation field consisting of about a hundred bubbles. The sinusoidal signal is direct electrical coupling with the driving transducer (operating at about $30 \mathrm{kHz}$ ). The large spikes that occur around $400 \mu \mathrm{s}$ are the result of the probe coming in contact with the cavitation field. Note that these spikes are positive; we can also obtain negative spikes (not shown here). We believe that these signals are directly a result of a bubble coming in contact with the probe.

Fig. 2. A differential probe is placed near a well-defined cavitation zone. Electrical coupling with the driving transducer gives rise to the observed sinusoidal variations, and correspond to the driving frequency. If the probe comes in contact with the cavitation zone, we observe electrical spikes superimposed on the sinusoidal signal.

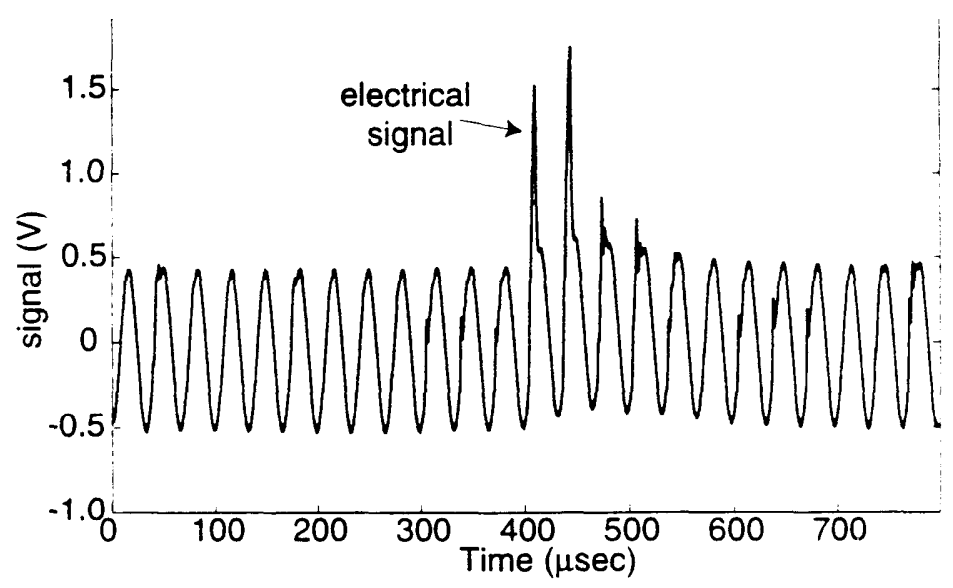

One possible mechanism for the observed spikes is that the collapsing bubble actually pushes against one of the probe tips, causing a change in capacitance between the differential probe tips. If the tips move closer together, one gets a unipolar, say, positive pulse. If the probe tips are pushed apart, the unipolar pulse becomes negative. We do not expect, nor have we observed. an electrical pulse from a single cavitating bubble, unless the bubble comes in direct contact with the probe tip. This evidence suggests that if there is an electrical pulse emanating from bubbles, it is weak. Another possibility is that charges from the bubble are being conducted to the probe tip during contact (it is known that bubble possess a negative charge).

Our goals for the first year also included performing near IR spectroscopy. Two cells have been designed for multiple bubble sonoluminescence (MBSL) spectroscopy experiments. The first cell is designed around a $20 \mathrm{kHz}$ acoustic horn with replaceable titanium tips from Sonics \& Materials (Fig. 3). The horn is squeezed into a stainless steel cell via O-rings and a compression ring, to seal the cell up to 100 psi for pressure experiments. The cell is thermostated by circulating coolant in a jacket, as well as flowing the cell fluid (at $4 \mathrm{~L} / \mathrm{min}$.) through a temperature control bath. Several ports are located on the cell for gas ports (one for headspace, another for bubbling), a pressure transducer, a thermocouple, a needle hydrophone, and a septum port for addition or withdrawal of samples. The total volume is approximately $80 \mathrm{~mL}$ with a $10 \mathrm{~mL}$ headspace. Directly opposite the horn tip is a $2 \mathrm{~cm}$ quartz window against which a fiber optic bundle is placed. Light collected through the fiber optic is imaged onto one of several detection systems, including a liquid nitrogen cooled Princeton CCD array, directly onto a room 
temperature PMT, or through an ISA double monochromator onto the PMT. This arrangement allows for great versatility and expandability.

The second cell is more useful for high frequency (from $100 \mathrm{kHz}$ to $1 \mathrm{MHz}$ ) experiments. The transducer is located at the bottom of 1.5 inch diameter teflon cylinder, and isolated from the sample matrix by a thin teflon sheet. Opposite the transducer is a $2 \mathrm{~cm}$ window in a stainless steel lid through which light is collected in the same manner as the above cell. Initial experiments in detecting NIR and IR light have been inconclusive. Further optimization may be needed to give more conclusive results.

\section{$20 \mathrm{kHz}$ MBSL Instrument Layout}

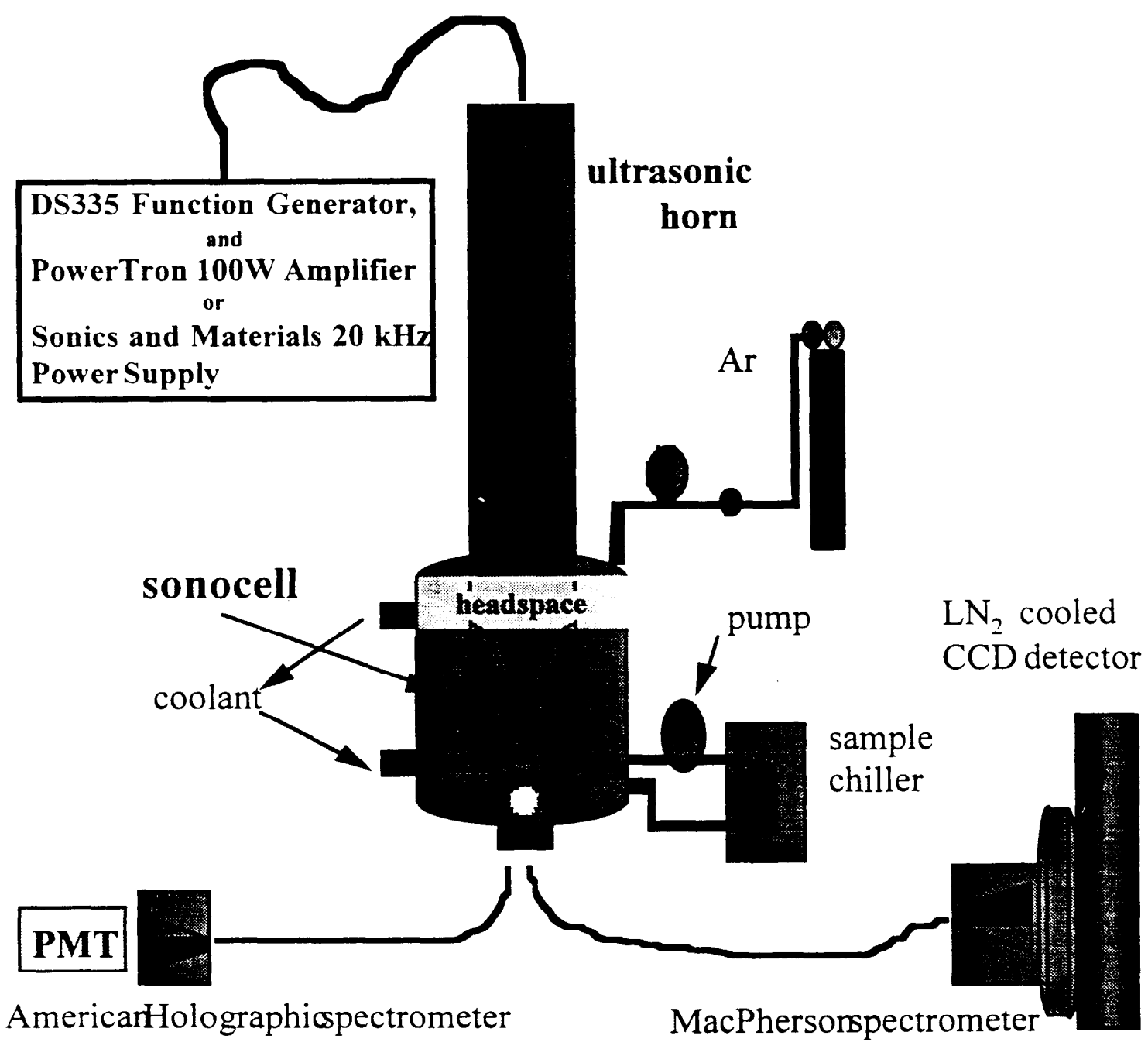

Fig. 3. Experimental apparatus for spectroscopy studies. This system allows us to change gas. liquid, and static (or hydraulic) pressure, at a constant temperature. 
An important factor in achieving good spectral results and efficient sonochemistry for MBSL is efficient cavitation with strong light output. Much of our progress in this area to date involves increasing the sonoluminescence output from our systems. Several techniques have been designed with this in mind. First, it has been observed that an increase of up to two orders of magnitude may be achieved by simply increasing the static pressure over the cavitating solution (see Fig. 4). With aqueous solutions a bimodal increase in light emission occurs with a local maximum at $30 \mathrm{psi}$. and a global maximum at $50 \mathrm{psi}$. With organic systems (such as heptane) a single maximum is observed at approximately $40 \mathrm{psi}$. which is 100 times brighter than at atmospheric pressure. The maximum in intensity is not well understood, since it is well known that increasing the ambient pressure should make cavitation more difficult, and thereby decrease the overall intensity from MBSL. We note that the decreasing intensity that we observe above 50 psi is probably due to the difficulty in generating cavitation at high static pressures. We are in the process of determining the corresponding effects from single cavitation bubbles.

Fig. 4. In this figure we show how the light intensity from MBSL varies as the static pressure is quasi-statically changed from ambient conditions of $1 \mathrm{~atm}$ (15 psi) to 4 atm (60 psi). We are able to achieve large increases in the total light output from many systems, in this particular case, heptane. The peak in intensity is not well understood, since it is well known that increasing the ambient pressure should make cavitation more difficult, and thereby decrease the overall intensity from MBSL.

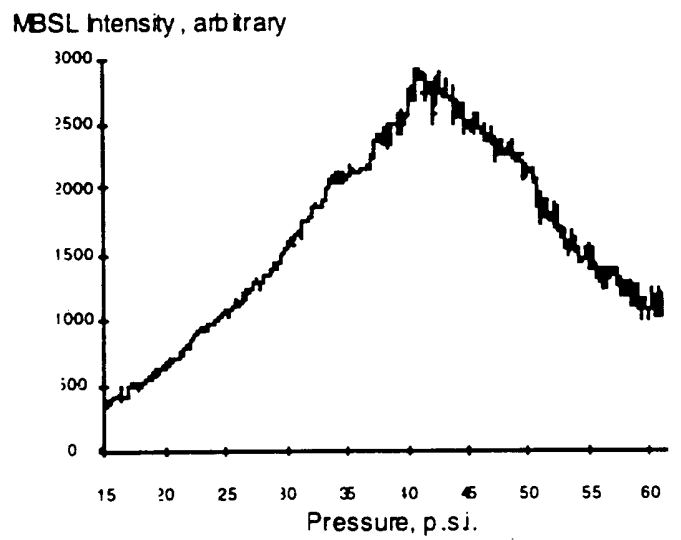

Another one to two orders of magnitude in intensity may be achieved by something that has come to be known in our labs as pressure jumping. In a typical experiment the pressure is quickly increased from atmospheric pressure to somewhere between 1.5 and 3 atmospheres. The jump occurs in approximately 1 second and is limited by the equilibrium time of flow from a 3 liter gas reservoir between the argon tank and the cell. The result of such an experiment is an immediate increase in sonoluminescence intensity, followed by a period of unstable, yet comparable intensity, ending with a return of the light level to the baseline level for that given pressure (see Fig. 5). This period of enhanced cavitation may last from 10 seconds to several minutes. depending on the solution, analytes, temperature, and final pressure. Similar results have been observed with frequency scanning and jumping, and remains to be further explored. So far, we have performed experiments in aqueous solutions of salts, with low concentrations of organics. organic solutions with low concentrations of water, and with pure organic systems. Each system has a unique set of physical properties that must be incorporated into a model for maximizing sonoluminescence output. From these experiments a paper has been submitted to Physical Review E titled "Cooperative Effects in Sonoluminescence." We feel that these results are very promising for generating more intense cavitation for liquid waste remediation. Although we have yet to fully understand the results, our coordinating work with a single-bubble cavitation system may shed light onto this matter. At present, we do not observe a similar behavior from singlebubble systems. 
Fig. 5. An abrupt pressure jump (from 1546 psi) is initiated in n-heptane. The total SL intensity increases by a factor of I6 in this case. We added trace amounts of thiophene, a heterocyclic, since it is well known to be very difficult to destroy with high intensity ultrasound. By increasing the cavitation intensity, here monitored as light production, we may be able to help degrade cyclic hydrocarbons in a more efficient manor.

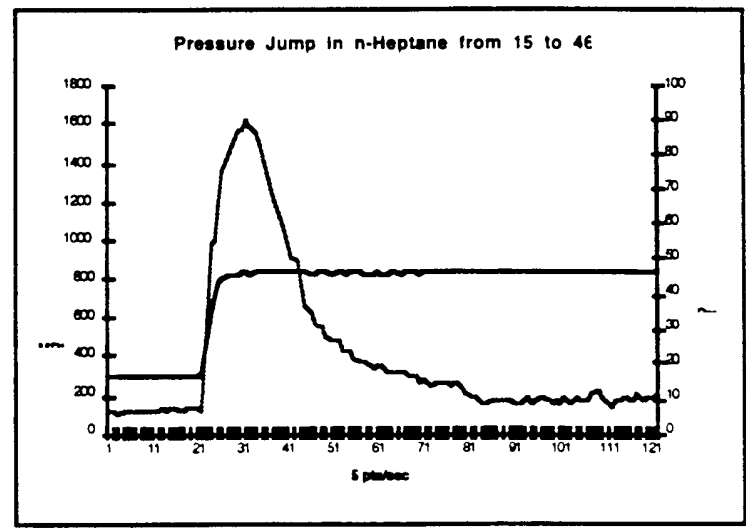

Finally, our expenses for the first year have been mostly salaries. This was anticipated since we had many pieces of equipment already in place to perform the initial experiments. Total expenses to date are approximately $\$ 120,000$. A full report of our expenses will follow, as outlined in the yearly expense report procedures. 